\title{
Transfer from serial to continuous paired-associate learning'
}

ROBERT G. CROWDER, DUGALD D. CHISHOLM AND DAVID A. FELL

Thirty-six Ss learned a serial list of words and were then tested on a paired-associate task involving short-term retention of once-presented pairs. When critical pairs on the transfer task had been derived from forward adjacent members of the serial list, performance significantly exceeded that for pairs which had not appeared on the serial list or which had been nonadjacent on the serial list.

The sequential hypothesis of serial verbal learning has been questioned recently by studies showing no positive transfer between serial and paired-associate lists when pairs in the latter had served as adjacent elements of the former (see review of Jensen \& Rohwer, 1965). The failure to obtain such transfer would not logically exclude the validity of the sequential hypothesis, however, if there should be reason to suspect rate of paired-associate list learning as a sensitive transfer task. The purpose of the present study was to determine whether a modified paired-associate task would reveal transfer from prior sequential associations.

The transfer task used here was devised by Peterson, Saltzman, Hillner, \& Land (1962) for studying shortterm retention of paired-associates and may be designated Continuous Paired-Associate (CPA) learning. In the CPA task, $S$ is exposed to a continuous, interlocking sequence of presentations and tests of verbal pairs. A given pair is presented only once and then tested once, later in the sequence, by exposure of the stimulus term alone; retention intervals are defined by the number of events (presentations of new pairs or tests) intervening between learning and recall of the pair in question. Since repetition is known to raise the short-term forgetting function (Hellyer, 1962) it was reasoned that if sequential associations are formed in serial learning, facilitation would be apparent in derived CPA items.

\section{Method}

The serial and CPA tasks were related according to one of three transfer conditions (12 Ss in each group). The Adjacent (Adj) group received as critical pairs in CPA learning items which hadbeen in adjacent positions (forward direction) of the serial list; the Non Adjacent (N Adj) group received pairs which had been members of the serial list, but separated by at least eight other items; and the Control (C) groups received no words in common on the two tasks. These three conditions combined with four retention intervals on the CPA task (within-Ss) yielded a mixed factorial design with 12 treatment combinations. All Ss received exactly the same CPA list; thus, variation was imposed through the nature of the prior serial lists. There were eight critical CPA pairs, two tested after one intervening event, two after two intervening events, two after four, and two after eight. Since all CPA events occurred at a $2 \mathrm{sec}$. rate, these specifications define retention intervals of $2,4,8$, and $16 \mathrm{sec}$.

All terms in both tasks were non-homophonic, fourletter, English nouns occurring at least $100 /$ million times in the Thorndike-Lorge count.

The CPA list contained 22 events, of which the first three were presentations of buffer pairs, designed to reduce possible primacy effects, but never tested. Three more buffer pairs, also never tested, were necessary elsewhere in the list to permit proper spacing of the critical pairs. The eight critical pairs accounted for the remaining 16 events with two instances of each of the four retention intervals, balanced as well as possible for serial position within the CPA list.

The Ss in the Adj group learned one of four (three Ss each) versions of the serial list for their first task. All four were 18 items long and shared (a) the initial two words, which were buffers, and (b) the sequence of words within pairs destined to appear in the CPA lists; however, the occurrence of these critical word pairs was balanced for serial position across the four versions of the serial list. Likewise, there were four 18-item serial lists learned by subgroups of the N Adj group. The same two buffer words began the list and in each version the critical pairs were separated by eight intervening words (except for separations of six and ten in one list). Words later to appear as response terms in CPA learning were balanced for serial position across the four $\mathrm{N}$ Adj serial lists. Group $\mathrm{C}$ learned a list of 18 words which bore no relation to the subsequent CPA task.

All materials were typed directly on a semi-opaque filmstrip base and delivered by a Graflex Compact filmstrip projector. Serial lists were learned by the recall method, with $2 \mathrm{sec}$. presentation speed and vocal recall. On test trials $\mathrm{S}$ was given a grid with 18 horizontal boxes and asked to point successively to each box as he recalled the elements of the list. Upon reaching criterion (two perfect recall trials), $\mathrm{S}$ was asked to remain and participate in a brief second experiment; nothing was said to indicate any particular relationship between the serial and CPA tasks. Instructions were then given on the procedures for CPA lists, and $S$ was given a short practice list involving pairs of arabic numbers. The CPA list was then administered and $\mathrm{S}$ was excused. 
The Ss were 36 Yale undergraduates who volunteered in order to satisfy a course requirement; they were assigned to groups on a rotating basis as they appeared. Results and Discussion

Groups Adj, $N$ Adj, and $C$ required means of 10.2, 6.3 , and 9.3 trials, respectively, to reach criterion on the prior serial learning task, $F=4.16, d f=2 / 33, p<.05$. Since four versions of each list were used in each group, this statistically significant variation in first-task learning speed was probably due to a fortuitous oversampling of fast learners in the $\mathrm{N}$ Adj group.

On the CPA task, total correct recalls (out of 24) at retention intervals of $1,2,4$, and 8 , respectively, were: for Adj Ss 19, 18, 7, and 12; for $N$ Adj Ss 15, 5, 2, and 5 ; and for C Ss 16, 6,2, and 6. A mixed factorial analysis of variance showed significant main effects of Transfer, $(\mathrm{F}=12.05, \mathrm{df}=2 / 33, \mathrm{p}<.01$, and Retention Interval, $\mathrm{F}=23.87, \mathrm{df}=3 / 99, \mathrm{p}<.01$ ), but a nonsignificant interaction $(F=1.42)$. Recall for the Adj group was nearly double that for the other two groups, whose performance was virtually identical. Since CPA items were completely confounded with retention interval in this study, no particular inference is warranted about the nonmonotonic forgetting function obtained. In all probability, idiosyncratic word pairings were responsible for these reversals.

The present results confirm data of Young (1961) in a similarly constructed study, but where the transfer task was paired-associate list learning, Young found tendencies for faster learning on the part of Adj Ss than controls, and for slower learning on the part of $\mathrm{N}$ Adj Ss; however, only the former result was statistically significant, and only for a partial criterion on the transfer task. Actually, predictions for $\mathrm{N}$ Adj groups are indeterminant in both of these studies, since beyond hypothesized negative transfer from serial learning, they should be subject to facilitative effects of response familiarization. Moreover, in the present study, CPA recall for $\mathrm{N}$ Adj Ss is probably further contaminated by their demonstrated superiority in learning. An interesting sidelight on transfer effects in these data is afforded by within-group correlations between trials to learn the serial list and CPA recall. These coefficients, for Adj, $\mathrm{N}$ Adj, and $\mathrm{C}$ groups, respectively, were $+.207,-.623$, and +.163 , of which only the second was statistically significant $(p<.05)$. Given that Ss who took more trials to complete serial learning had relatively more experience with the critical words than faster serial learners, these correlations show dramatically the influence of transfer above and beyond effects of response familiarization.

These data suggest a virtue in using a variety of associative transfer tasks in testing the sequential hypothesis of serial learning. They are, of course, perfectly consistent with reports of early positive effects in paired-associate list learning (Jensen \& Rowher, 1965). Equally compatible with these results are data from the association method of testing for remote associations following serial learning (McGeoch \& Irion, 1952). It is perhaps premature to abandon completely sequential associations as a necessary (though probably not sufficient) ingredient of the theory of serial learning.

\section{References}

Hellyer, s. Supplementary report: Frequency of stimulus presentation and short-term decrement in recall. J. exp. Psychol., 1962, 64,650 .

Jensen, A. R., \& Rohwer, W. D. Jr. What is learned in serial learning? J. verbal Learn. verbal Behav., 1965, 4, 62-72.

McGeoch, J. A., \& Irion, A. L. The psychology of human learning. (2nd ed.) New York: Longmans Green, 1952.

Peterson, L. R., Saltzman, D., Hillner, K., \& Land, V. Recency and frequency in paired-associate learning. J. exp. Psychol., $1962,63,396-403$.

Young, R. K. The stimulus in serial verbal learning. Amer. J. Psychol., 1961, 74, 517-528.

\section{Note}

1. This research was conducted as part of the senior author's course in learning. Funds for purchase of equipment were supplied by Yale University. 